\title{
Damping and Pseudo-fermions
}

\author{
F. Bagarello \\ Dieetcam, Facoltà di Ingegneria, \\ Università di Palermo, I-90128 Palermo, Italy \\ e-mail: fabio.bagarello@unipa.it \\ home page: www.unipa.it \fabio.bagarello
}

\begin{abstract}
After a short abstract introduction on the time evolution driven by non self-adjoint hamiltonians, we show how the recently introduced concept of pseudo-fermion can be used in the description of damping in finite dimensional quantum systems, and we compare the results deduced adopting the Schrödinger and the Heisenberg representations.
\end{abstract}




\section{Introduction}

In a series of papers, [1]-8], we have considered two operators $a$ and $b$, with $b \neq a^{\dagger}$, acting on a Hilbert space $\mathcal{H}$, and satisfying the commutation rule $[a, b]=\mathbb{1}$. A nice functional structure has been deduced under suitable assumptions, and some connections with physics, and in particular with what is usually called quasi-hermitian quantum mechanics and with the technique of intertwining operators, have been established. Following Trifonov, [9], we have called pseudo-bosons (PB) the particle-like excitations associated to this structure. A similar analysis has also been carried out for what we have called nonlinear pseudo-bosons (NLPB) in [10]-[12], and most of the original results have been recovered also in this more general situation, where the main ingredient is not the commutation rule between $a$ and $b$ but their raising and lowering properties, when applied to two fixed biorthogonal bases. The analytical treatment of both PB and NLPB turns out to be particularly difficult in the case where regularity is lost, that is, see below, when these bases are not Riesz bases. In this case, in fact, the intertwining operators appearing in the game (whose square roots are metric operators in the sense of the literature on quasi-hermitian quantum mechanics, see [13, 14, 15] and references therein) turns out to be unbounded. For this reason, a large amount of mathematical care is required, and this makes the rigorous treatment of the physical system rather complicated, [12].

More recently, [16], we have introduced a similar extension for the canonical anticommutation relation (CAR), following an original idea by Trifonov and his collaborators, [17, also briefly considered in [18] and in [19]. The idea is very similar to the previous one: we consider again two operators $a$ and $b$, with $b \neq a^{\dagger}$, acting on a Hilbert space $\mathcal{H}$, and satisfying the anticommutation rule $\{a, b\}=\mathbb{1}$, as well as $a^{2}=b^{2}=0$. Of course, if $b=a^{\dagger}$ we go back to ordinary CAR. The functional structure that we deduce out of these operators is nice and friendly, since $\mathcal{H}$ is finite-dimensional. The related excitations are called pseudo-fermions, (PF). One of the most important features of $\mathrm{PF}$ is that all the problems arising when dealing with $\mathrm{PB}$, because of their (in general) unbounded nature, are now absent.

In this paper we focus on a particular aspect of PF, i.e. on their possible use in the analysis of damped quantum systems. More in details: in the description of damping in quantum optics certain finite dimensional, non self-adjoint, matrices are quite often used. These matrices are usually called effective hamiltonians. Solving the related Schrödinger equation, one sees that the wave-function of the system goes to zero for large $t$, at least if the parameters of the model are chosen properly. Already in [16] we have seen that one such a model, originally proposed in [20], can be easily written in terms of PF. However, we have not explored the damping 
features of that system, because that was not our major interest. Here, on the other hand, we focus our attention exactly on this aspect, and we work both in the Schrödinger and in the Heisenberg representations, showing that analogous conclusions can be deduced. We also extend our analysis to a higher-dimensional Hilbert space, which we will relate to a two-dimensional family of PF. For both these examples we deduce damping 1 .

The paper is organized as follows: in the next section we briefly discuss some basic facts on $\mathrm{PF}$ and on the time evolution in the Heisenberg representation, when this is driven by some non self-adjoint hamiltonian. This, we believe, is useful since not all the readers are familiar with this kind of time-evolution, and surely less readers are familiar with pseudo-fermions. Section III is devoted to our first, two-dimensional, example, while in Section IV we consider a four-dimensional, example. For both these examples we compare the well known Schrödinger point of view with the modified Heisenberg evolution, and we show that, not surprisingly, the same conclusions can be deduced. Particularly relevant for us is the fact that, after a somehow standard transformation of the effective hamiltonian, we will naturally driven to introduce pseudo-fermions in the game. Section V contains an abstract generalization of the approach, while our conclusions are given in Section VI.

\section{Pseudo-fermions and dynamics}

We begin this section by briefly reviewing the main definitions and results concerning PF in one dimension. The extension to higher dimensions will be discussed later on. The starting point is a modification of the CAR $\left\{c, c^{\dagger}\right\}=c c^{\dagger}+c^{\dagger} c=\mathbb{1},\{c, c\}=\left\{c^{\dagger}, c^{\dagger}\right\}=0$, between two operators, $c$ and $c^{\dagger}$, acting on a two-dimensional Hilbert space $\mathcal{H}$. The CAR are replaced here by the following rules:

$$
\{a, b\}=\mathbb{1}, \quad\{a, a\}=0, \quad\{b, b\}=0,
$$

where the interesting situation is when $b \neq a^{\dagger}$. These rules automatically imply that a non zero vector, $\varphi_{0}$, exists in $\mathcal{H}$ such that $a \varphi_{0}=0$, and that a second non zero vector, $\Psi_{0}$, also exists in $\mathcal{H}$ such that $b^{\dagger} \Psi_{0}=0$, [16].

Under these conditions it is possible to recover similar results as those for PB. In particular, we first introduce the following non zero vectors

$$
\varphi_{1}:=b \varphi_{0}, \quad \Psi_{1}=a^{\dagger} \Psi_{0}
$$

\footnotetext{
${ }^{1}$ It might be worth stressing that we are not claiming here that PF are always relevant to explain damping.
} 
as well as the non self-adjoint operators

$$
N=b a, \quad \mathfrak{N}=N^{\dagger}=a^{\dagger} b^{\dagger} .
$$

We further introduce the self-adjoint operators $S_{\varphi}$ and $S_{\Psi}$ via their action on a generic $f \in \mathcal{H}$ :

$$
S_{\varphi} f=\sum_{n=0}^{1}\left\langle\varphi_{n}, f\right\rangle \varphi_{n}, \quad S_{\Psi} f=\sum_{n=0}^{1}\left\langle\Psi_{n}, f\right\rangle \Psi_{n} .
$$

Hence we get the following results, whose proofs are straightforward and will not be given here:

1.

$$
a \varphi_{1}=\varphi_{0}, \quad b^{\dagger} \Psi_{1}=\Psi_{0} .
$$

2 .

$$
N \varphi_{n}=n \varphi_{n}, \quad \mathfrak{N} \Psi_{n}=n \Psi_{n},
$$

for $n=0,1$.

3. If the normalizations of $\varphi_{0}$ and $\Psi_{0}$ are chosen in such a way that $\left\langle\varphi_{0}, \Psi_{0}\right\rangle=1$, then

$$
\left\langle\varphi_{k}, \Psi_{n}\right\rangle=\delta_{k, n},
$$

for $k, n=0,1$.

4. $S_{\varphi}$ and $S_{\Psi}$ are bounded, strictly positive, self-adjoint, and invertible. They satisfy

$$
\begin{gathered}
\left\|S_{\varphi}\right\| \leq\left\|\varphi_{0}\right\|^{2}+\left\|\varphi_{1}\right\|^{2}, \quad\left\|S_{\Psi}\right\| \leq\left\|\Psi_{0}\right\|^{2}+\left\|\Psi_{1}\right\|^{2}, \\
S_{\varphi} \Psi_{n}=\varphi_{n}, \quad S_{\Psi} \varphi_{n}=\Psi_{n},
\end{gathered}
$$

for $n=0,1$, as well as $S_{\varphi}=S_{\Psi}^{-1}$. Moreover, the following intertwining relations

$$
S_{\Psi} N=\mathfrak{N} S_{\Psi}, \quad S_{\varphi} \mathfrak{N}=N S_{\varphi},
$$

hold.

The above formulas show that (i) $N$ and $\mathfrak{N}$ behave as fermionic number operators, having eigenvalues 0 and 1; (ii) their related eigenvectors are respectively the vectors of $\mathcal{F}_{\varphi}=\left\{\varphi_{0}, \varphi_{1}\right\}$ and $\mathcal{F}_{\Psi}=\left\{\Psi_{0}, \Psi_{1}\right\}$; (iii) $a$ and $b^{\dagger}$ are lowering operators for $\mathcal{F}_{\varphi}$ and $\mathcal{F}_{\Psi}$ respectively; (iv) $b$ and $a^{\dagger}$ are rising operators for $\mathcal{F}_{\varphi}$ and $\mathcal{F}_{\Psi}$ respectively; (v) the two sets $\mathcal{F}_{\varphi}$ and $\mathcal{F}_{\Psi}$ are 
biorthonormal; (vi) the well-behaved ${ }^{2}$ operators $S_{\varphi}$ and $S_{\Psi}$ maps $\mathcal{F}_{\varphi}$ in $\mathcal{F}_{\Psi}$ and viceversa; (vii) $S_{\varphi}$ and $S_{\Psi}$ intertwine between operators which are not self-adjoint, in the very same way as they do for PB.

We refer to [16] for further remarks and consequences of these definitions. In particular, for instance, it is shown that $\mathcal{F}_{\varphi}$ and $\mathcal{F}_{\Psi}$ are automatically Riesz bases for $\mathcal{H}$, and the relations between fermions and PF are discussed.

\section{II.1 The algebraic dynamics}

In [20] a non self-adjoint effective hamiltonian is considered in the description of the time evolution of the wave-function of a two-dimensional quantum system. The resulting dynamics shows a decay of this wave-function. This is just a single reference where such an approach is considered. Other references are, for instance, [17], 21] and, for more abstract considerations, [22]. The starting point is always the same: a Schrödinger equation

$$
i \dot{\Psi}(t)=H_{e f f} \Psi(t)
$$

where $H_{\text {eff }} \neq H_{\text {eff }}^{\dagger}$. Actually, in [20] and [17], $H_{\text {eff }}$ is assumed to be pseudo-hermitian. We will not consider this extra assumption in the first part of this section. It is worth stressing here that formula (2.11) should be considered as an assumption in itself, since in standard quantum mechanics the Schrödinger equation is usually (if not always) assumed to hold for a self-adjoint hamiltonian. Of course, if $H_{\text {eff }}$ does not depend explicitly on time, the formal solution of (2.11) is $\Psi(t)=e^{-i H_{e f f} t} \Psi(0)$. It is clear that, if $H_{e f f}$ is a finite-dimensional matrix, this exponential can be defined using, for instance, a norm convergent series:

$$
e^{-i H_{e f f} t}:=\|.\|-\sum_{k=0}^{\infty} \frac{(-i t)^{k}}{k !} H_{e f f}^{k} .
$$

On the other hand, if $H_{e f f}$ is an unbounded operator, defining $e^{-i H_{e f f} t}$ is quite a delicate task, since we cannot even use the spectral theorem. We will not consider this aspect of the theory here, since we are concerned only with finite matrices. Solving (2.11) means that we are adopting the Schrödinger representation. To move to the Heisenberg representation, we assume, as it is always done in ordinary quantum mechanics, that the time evolution of the mean values of the observables do not depend on the representation chosen. In other words,

\footnotetext{
${ }^{2}$ i.e. self-adjoint, bounded, invertible, with bounded inverse
} 
if $X$ is an observable of our physical system, calling $X_{\text {eff }}(t)$ its time evolution driven by $H_{e f f}$, we require that

$$
\langle\Psi(t), X \Psi(t)\rangle=\left\langle\Psi(0), X_{e f f}(t) \Psi(0)\right\rangle .
$$

Since this equality must hold for all possible choices of $\Psi(0)$, we deduce that

$$
X_{e f f}(t):=e^{i H_{e f f}^{\dagger} t} X e^{-i H_{e f f} t} .
$$

This is the time evolution of $X$ in the Heisenberg representation. Notice that, if $H_{\text {eff }}=H_{\text {eff }}^{\dagger}=$ : $H$, we go back to the usual formula and to the unitary evolution, $X(t):=e^{i H t} X e^{-i H t}$. Notice also that, while $(X Y)(t)=X(t) Y(t),(X Y)_{\text {eff }}(t) \neq X_{\text {eff }}(t) Y_{\text {eff }}(t)$ : the time evolution in (2.12) is not an automorphism of the set of the observables of the system. Another interesting feature of (2.12) is that this time evolution is stable under the adjoint: $\left(X_{\text {eff }}(t)\right)^{\dagger}=\left(X^{\dagger}\right)_{\text {eff }}(t)$.

Also, it is clear that the Heisenberg equation of motion for $X_{\text {eff }}(t)$ should be modified. For that, let us define the following effective commutator between two generic operators $A$ and $B$ :

$$
[A, B]_{e f f}:=A B-B^{\dagger} A \text {. }
$$

The effective commutator satisfies, for example, the following equality: given three generic operators $A, B$ and $C$, then

$$
[A B, C]_{e f f}=A[B, C]_{e f f}+[A, C]_{e f f} B+A\left(C^{\dagger}-C\right) B
$$

which reduces to the standard formula when $C=C^{\dagger}$, but not in general. Also, not surprisingly, the Jacobi identity ceases to be true for $[., .]_{\text {eff }}$. Still we have $[A, B]_{\text {eff }}^{\dagger}=-\left[A^{\dagger}, B\right]_{\text {eff }}$.

The differential equation for $X_{\text {eff }}(t)$ is the following:

$$
i \frac{d}{d t} X_{e f f}(t)=\left[X_{e f f}(t), H_{e f f}\right]_{e f f}=e^{i H_{e f f}^{\dagger} t}\left[X, H_{e f f}\right]_{e f f} e^{-i H_{e f f} t}
$$

which returns the standard equation if $H_{\text {eff }}=H_{\text {eff }}^{\dagger}$. This formula shows, among other things, that an integral of motion, $Z$, is not an operator commuting with $H_{\text {eff }}$. What $Z$ has to satisfy is clearly the following requirement: $\left[Z, H_{e f f}\right]_{e f f}=0$. In fact, if this is true, then $\dot{Z}_{e f f}(t)=0$.

In [21] the authors have considered a slightly different point of view, splitting $H_{\text {eff }}$ into two parts, both self-adjoint: $H_{e f f}=\frac{1}{2}\left(H_{e f f}+H_{e f f}^{\dagger}\right)-i \frac{1}{2 i}\left(H_{e f f}^{\dagger}-H_{e f f}\right)$. In this way, rather than introducing an effective commutator, they deduce a differential equation for $X_{\text {eff }}(t)$ in which both a commutator and an anti-commutator appear. 
Something more can be said if $H_{e f f}$ is crypto-hermitian, see [11, i.e. if a positive selfadjoint, time-independent, operator $\Theta$ exists, bounded with bounded inverse, such that $H_{\text {eff }}=$ $\Theta^{-1} H_{e f f}^{\dagger} \Theta$. In this case we get the following:

$$
\Theta^{-1} X_{e f f}(t)=e^{i H_{e f f} t}\left(\Theta^{-1} X\right) e^{-i H_{e f f} t},
$$

and the differential equation is

$$
\frac{d}{d t}\left(\Theta^{-1} X_{e f f}(t)\right)=i e^{i H_{e f f} t}\left[H_{e f f}, \Theta^{-1} X\right] e^{-i H_{e f f} t} .
$$

Let us remark that here, first of all, the commutator is the standard one. Also, at a first sight the time evolution for $\Theta^{-1} X$ might appear as an automorphism. Nevertheless, it is not hard to check that this is not true, exactly because of the presence of $\Theta^{-1}$. In fact, $\Theta^{-1} X_{\text {eff }}(t)$ is, in general, different from $\left(\Theta^{-1} X\right)_{e f f}(t)$. A simple case when they coincide is when $H_{\text {eff }}$ is self-adjoint. The essential reason why formulas above look interesting is because only $H_{\text {eff }}$ appears, and not $H_{\text {eff }}^{\dagger}$. In particular, the differential equation shows that $X_{\text {eff }}(t)$ is constant when $H_{\text {eff }}$ commutes not with $X$ but with $\Theta^{-1} X$. This is the formula which replaces, in this particular case, the one deduced above, $\left[X, H_{\text {eff }}\right]_{\text {eff }}=0$.

Because of the properties of $\Theta$, we know that $\Theta^{ \pm 1 / 2}$ can be defined, and $h:=\Theta^{1 / 2} H_{\text {eff }} \Theta^{-1 / 2}$ is a self-adjoint operator: $h=h^{\dagger}$. Let us now introduce the following automorphism of $B(\mathcal{H})$, the set of the bounded operators on $\mathcal{H}: j_{\Theta}(x):=\Theta^{-1 / 2} x \Theta^{1 / 2}$. This map is invertible, and it is clear that $h=j_{\Theta}^{-1}\left(H_{e f f}\right)$. If we further define the following standard Heisenberg evolution, $\alpha^{t}(X):=e^{i h t} X e^{-i h t}, X \in B(\mathcal{H})$, which has all the usual properties of the time evolution for systems driven by self-adjoint hamiltonians, we deduce that

$$
X_{e f f}(t)=j_{\Theta}^{-1}\left(\alpha^{t}\left(j_{\Theta}(X)\right)\right)
$$

which has a first interesting consequence: this effective time evolution coincides with $\alpha^{t}$ if this latter commutes with $j_{\Theta}$. Moreover, the interpretation is the following: to find $X_{e f f}(t)$, we have first to map $X$ into $j_{\Theta}(X)$. Then we let this operator evolve using $h$, and finally we transform back this result, using $j_{\Theta}^{-1}$; therefore, the effective time evolution appears to be the composition of these three maps. 


\section{An example from the literature}

The first example is taken by [17, 20], and its relation with PF was already partly considered in [16]. The starting point is the Schrödinger equation

$$
i \dot{\Psi}(t)=H_{e f f} \Psi(t), \quad \text { with } \quad H_{e f f}=\frac{1}{2}\left(\begin{array}{cc}
-i \gamma_{a} & v \\
\bar{v} & -i \gamma_{b}
\end{array}\right),
$$

where $\gamma_{a}, \gamma_{b}>0$ and $v \in \mathbb{C}$.

\section{III.1 Schrödinger representation}

With a simple change of variable $\Phi(t)=e^{\Gamma t} \Psi(t), \Gamma=\frac{1}{2}\left(\gamma_{a}+\gamma_{b}\right)$, we deduce that $i \dot{\Phi}(t)=H \Phi(t)$, where

$$
H=i \Gamma \mathbb{1}_{2}+H_{e f f}=\left(\begin{array}{cc}
-i \gamma & v \\
\bar{v} & i \gamma
\end{array}\right), \quad \Phi(t)=\left(\begin{array}{c}
\Phi_{0}(t) \\
\Phi_{1}(t)
\end{array}\right) .
$$

Here $\mathbb{1}_{2}$ is the two-by-two identity matrix, and $\gamma=\frac{1}{2}\left(\gamma_{a}-\gamma_{b}\right)$. The differential equations for $\Phi_{0}(t)$ and $\Phi_{1}(t)$ are easily deduced. Let us introduce $\Omega:=|v|^{2}-\gamma^{2}$. Then

$$
\left\{\begin{array}{l}
\ddot{\Phi}_{0}(t)=-\Omega \Phi_{0}(t) \\
\ddot{\Phi}_{1}(t)=-\Omega \Phi_{1}(t) .
\end{array}\right.
$$

If $\Omega=0$ then the functions $\Phi_{0}(t)$ and $\Phi_{1}(t)$ are linear in $t$, so that

$$
\Psi(t)=e^{-\Gamma t}\left(\begin{array}{c}
\Phi_{0}(t) \\
\Phi_{1}(t)
\end{array}\right)=\left(\begin{array}{c}
e^{-\left(\gamma_{a}+\gamma_{b}\right) \frac{t}{2}}\left(A_{0}+B_{0} t\right) \\
e^{-\left(\gamma_{a}+\gamma_{b}\right) \frac{t}{2}}\left(A_{1}+B_{1} t\right)
\end{array}\right),
$$

where $A_{0}, A_{1}, B_{0}$ and $B_{1}$ are fixed by the initial conditions. In particular, if $\Psi(0)=\left(\begin{array}{l}\varphi_{0} \\ \varphi_{1}\end{array}\right)$, we find that $A_{0}=\varphi_{0}, A_{1}=\varphi_{1}, B_{0}=-\gamma \varphi_{0}-i v \varphi_{1}$ and $B_{1}=\gamma \varphi_{1}-i \bar{v} \varphi_{0}$.

The norm of $\Psi(t)$ is simply the norm in $\mathcal{H}=\mathbb{C}^{2}$. Therefore

$$
\|\Psi(t)\|^{2}=e^{-t\left(\gamma_{a}+\gamma_{b}\right)}\left(\left|A_{0}+B_{0} t\right|^{2}+\left|A_{1}+B_{1} t\right|^{2}\right),
$$

and this squared norm goes to zero when $t \rightarrow \infty$.

Let us consider now the case in which $\Omega>0$. In this case the solution can be written as

$$
\Psi(t)=e^{-\left(\gamma_{a}+\gamma_{b}\right) \frac{t}{2}}\left(\begin{array}{c}
A_{0} \cos (\sqrt{\Omega} t)+B_{0} \sin (\sqrt{\Omega} t) \\
A_{1} \cos (\sqrt{\Omega} t)+B_{1} \sin (\sqrt{\Omega} t)
\end{array}\right)
$$


where, again, $A_{0}, A_{1}, B_{0}$ and $B_{1}$ are fixed by the initial conditions: $A_{0}=\varphi_{0}, A_{1}=\varphi_{1}, B_{0}=$ $\frac{1}{\sqrt{\Omega}}\left(-\gamma \varphi_{0}-i v \varphi_{1}\right)$ and $B_{1}=\frac{1}{\sqrt{\Omega}}\left(\gamma \varphi_{1}-i \bar{v} \varphi_{0}\right)$. In this case $\|\Psi(t)\|^{2}$ is the product of a decaying exponential and an oscillating function. More explicitly,

$$
\|\Psi(t)\|^{2}=e^{-t\left(\gamma_{a}+\gamma_{b}\right)}\left(\left|A_{0} \cos (\sqrt{\Omega} t)+B_{0} \sin (\sqrt{\Omega} t)\right|^{2}+\left|A_{1} \cos (\sqrt{\Omega} t)+B_{1} \sin (\sqrt{\Omega} t)\right|^{2}\right),
$$

which decays to zero independently of the particular values of $\varphi_{0}$ and $\varphi_{1}$.

Let us finally consider the case in which $\Omega<0$. In this case the solution can be written as

$$
\Psi(t)=e^{-\left(\gamma_{a}+\gamma_{b}\right) \frac{t}{2}}\left(\begin{array}{c}
A_{0} \exp (\sqrt{|\Omega|} t)+B_{0} \exp (-\sqrt{|\Omega|} t) \\
A_{1} \exp (\sqrt{|\Omega|} t)+B_{1} \exp (-\sqrt{|\Omega|} t)
\end{array}\right) .
$$

Here, $A_{0}, A_{1}, B_{0}$ and $B_{1}$ are related to the initial conditions as follows:

$$
\begin{aligned}
& A_{0}=\frac{1}{2}\left(\varphi_{0}+\frac{1}{\sqrt{|\Omega|}}\left(-\gamma \varphi_{0}-i v \varphi_{1}\right)\right), \quad B_{0}=\frac{1}{2}\left(\varphi_{0}+\frac{1}{\sqrt{|\Omega|}}\left(\gamma \varphi_{0}+i v \varphi_{1}\right)\right), \\
& A_{1}=\frac{1}{2}\left(\varphi_{1}+\frac{1}{\sqrt{|\Omega|}}\left(\gamma \varphi_{1}-i \bar{v} \varphi_{0}\right)\right), \quad B_{1}=\frac{1}{2}\left(\varphi_{1}+\frac{1}{\sqrt{|\Omega|}}\left(-\gamma \varphi_{1}+i \bar{v} \varphi_{0}\right)\right) .
\end{aligned}
$$

In this case it is not completely clear that $\|\Psi(t)\|$ goes to zero for increasing $t$. However, to get such a conclusion, it is enough to consider the asymptotic behavior of $e^{-\left(\gamma_{a}+\gamma_{b}\right) t} e^{2 \sqrt{|\Omega|} t}$, which is the only dangerous contribution appearing in $\|\Psi(t)\|^{2}$. The conclusion is that $\|\Psi(t)\| \rightarrow 0$, when $t \rightarrow \infty$, if $2 \sqrt{|\Omega|}<\gamma_{a}+\gamma_{b}$, which is always satisfied.

The outcome of this analysis is, therefore, the following: the wave-function $\Psi(t)$ solving the Schrödinger equation (3.1) goes to zero independently of the value of $\Omega$.

\section{III.2 Heisenberg representation}

It is easy to see that $\Omega$ is related to the eigenvalues of $H$, which are $\lambda_{ \pm}:= \pm \sqrt{|v|^{2}-\gamma^{2}}$. Hence $\lambda_{+}=\lambda_{-}=0$ if $\Omega=0$, while $\lambda_{ \pm}:= \pm \sqrt{\Omega}$ when $\Omega>0$. Finally, $\lambda_{ \pm}:= \pm i \sqrt{|\Omega|}$ when $\Omega<0$. In all these cases the eigenstates of $H$ can be written as

$$
\eta_{+}=\left(\begin{array}{c}
\frac{1}{\bar{v}}(-i \gamma+\sqrt{\Omega}) \\
1
\end{array}\right), \quad \eta_{-}=\left(\begin{array}{c}
-\frac{1}{\bar{v}}(i \gamma+\sqrt{\Omega}) \\
1
\end{array}\right) .
$$

In fact, $H \eta_{ \pm}=\lambda_{ \pm} \eta_{ \pm}$. Moreover, in general $\eta_{+}$and $\eta_{-}$are not orthogonal: $\left\langle\eta_{+}, \eta_{-}\right\rangle=$ $\frac{2 \gamma}{|v|^{2}}(\gamma-i \sqrt{\Omega})$, which is zero only if $\gamma=0\left(H=H^{\dagger}\right)$ or if $\gamma=i \sqrt{\Omega}\left(H=-H^{\dagger}\right)$. Due to the relation between $H$ and $H_{\text {eff }}$ we also find that

$$
H_{e f f} \eta_{ \pm}=E_{ \pm} \eta_{ \pm}, \quad E_{ \pm}=-\frac{i}{2}\left(\gamma_{a}+\gamma_{b}\right) \pm \sqrt{\Omega}
$$


In [16] we have analyzed, in connection with PF, the following non self-adjoint operator,

$$
\tilde{H}=\frac{1}{2}\left(\begin{array}{cc}
-i \delta & \bar{\omega} \\
\omega & i \delta
\end{array}\right),
$$

which coincides with our $H$ simply taking $\delta=2 \gamma$ and $\omega=2 \bar{v}$. As in [16], it is therefore possible to introduce two operators $a$ and $b$, such that $\{a, b\}=\mathbb{1}, a^{2}=b^{2}=0$, and $H=\Omega\left(b a-\frac{1}{2} \mathbb{1}\right)$. Introducing also the number operators $N=b a$ and $\mathfrak{N}=N^{\dagger}$, and assuming, just to fix the ideas, that $\Omega>0$, we can write $H=\Omega\left(N-\frac{1}{2} \mathbb{1}\right)$ and $H^{\dagger}=\Omega\left(\mathfrak{N}-\frac{1}{2} \mathbb{1}\right)$. Now, we want to recover here the same damping we have found working in Schrödinger representation. For that, it is natural to consider the time evolution of the number operators $N$ and $\mathfrak{N}$, which should be computed as in (2.12):

$$
N_{\text {eff }}(t)=e^{i H_{\text {eff }}^{\dagger} t} N e^{-i H_{\text {eff }} t}, \quad \mathfrak{N}_{\text {eff }}(t)=e^{i H_{\text {eff }}^{\dagger} t} \mathfrak{N} e^{-i H_{\text {eff }} t}=\left(N_{\text {eff }}(t)\right)^{\dagger} .
$$

Due to the relation between $H_{\text {eff }}$ and $H, H_{\text {eff }}=H-i \Gamma \mathbb{1}$, and to the above expressions for $H$ and $H^{\dagger}$, we find that, for each observable $X$,

$$
X_{e f f}(t)=e^{-2 \Gamma t} e^{i \Omega \mathfrak{N} t} X e^{-i \Omega N t} .
$$

Therefore, recalling that the anticommutation rules imply that $N^{2}=N, \mathfrak{N}^{2}=\mathfrak{N}$, and so on, we find that

$$
N_{e f f}(t)=e^{-2 \Gamma t}\left(N e^{-i \Omega t}+\mathfrak{N} N\left(1-e^{-i \Omega t}\right)\right),
$$

while $\mathfrak{N}_{\text {eff }}(t)$ is simply its adjoint. Then, if we estimate the norm of $N_{\text {eff }}(t)$, it is trivial to deduce that $\left\|N_{\text {eff }}(t)\right\| \leq 3 e^{-2 \Gamma t}$, which goes to zero when $t$ diverges. Hence, as expected, we recover damping also in Heisenberg picture, with no need of introducing extra assumptions on the parameters of the system. More connections with PF are discussed in [16].

\section{A two-dimensional example}

We are going now to repeat the same analysis considering the following four-by-four effective hamiltonian:

$$
H_{e f f}=\frac{i}{\alpha-\beta}\left(\begin{array}{cccc}
0 & -\alpha \beta\left(\omega_{1}-\omega_{2}\right) & 0 & 0 \\
\omega_{1}-\omega_{2} & -(\alpha+\beta)\left(\omega_{1}-\omega_{2}\right) & 0 & 0 \\
-\omega_{2} & \alpha \omega_{2} & \beta \omega_{2}-\alpha \omega_{1} & 0 \\
-\beta \omega_{2} & \beta^{2} \omega_{2} & 0 & \alpha \omega_{2}-\beta \omega_{1}
\end{array}\right) \text {, }
$$

where $\alpha \neq \beta$ and $\omega_{1}>\omega_{2}>0$. It is evident that $H_{\text {eff }} \neq H_{\text {eff }}^{\dagger}$. We will show that, also for this hamiltonian, a decay can be deduced. 


\section{IV.1 Schrödinger representation}

As before, we want to solve first the Schrödinger equation $i \dot{\Psi}(t)=H_{e f f} \Psi(t)$ and check whether damping is deduced, and in which conditions. Even if $H_{e f f}$ has no direct physical interpretation, still it is interesting, since, with a simple transformation, we can transform the original Schrödinger equation into a new one, with a traceless hamiltonian $H$, which is also not selfadjoint. This is very close to what was done, for instance, in [17, 16, 20], and in the previous section. For that it is enough to proceed as in the previous example, defining $\Phi(t)=e^{\Gamma t} \Psi(t)$, $H=i \Gamma \mathbb{1}_{4}+H_{e f f}$, and $\Gamma=\frac{\alpha+\beta}{2(\alpha-\beta)}\left(\omega_{1}-\omega_{2}\right)$, where $\mathbb{1}_{4}$ is the identity matrix in $\mathcal{H}=\mathbb{C}^{4}$. Then we get

$$
i \dot{\Phi}(t)=H \Phi(t)
$$

where

$H=\frac{i}{\alpha-\beta}\left(\begin{array}{cccc}\frac{1}{2}(\alpha+\beta)\left(\omega_{1}-\omega_{2}\right) & -\alpha \beta\left(\omega_{1}-\omega_{2}\right) & 0 & 0 \\ \omega_{1}-\omega_{2} & -\frac{1}{2}(\alpha+\beta)\left(\omega_{1}-\omega_{2}\right) & 0 & 0 \\ -\omega_{2} & \alpha \omega_{2} & -\frac{1}{2}(\alpha-\beta)\left(\omega_{1}+\omega_{2}\right) & 0 \\ -\beta \omega_{2} & \beta^{2} \omega_{2} & 0 & \frac{1}{2}(\alpha-\beta)\left(\omega_{1}+\omega_{2}\right)\end{array}\right)$,

and

$$
\Phi(t)=\left(\begin{array}{c}
\Phi_{0}(t) \\
\Phi_{1}(t) \\
\Phi_{2}(t) \\
\Phi_{3}(t)
\end{array}\right)
$$

As in our first example, the matrix $H$ is traceless. The related Schrödinger equation of motion produces, first of all, $\ddot{\Phi}_{0}(t)=\Omega \Phi_{0}(t)$, where $\Omega:=\frac{1}{2}\left|\omega_{1}-\omega_{2}\right|$. Then $\Phi_{0}(t)=\tilde{A}_{0} e^{\Omega t}+\tilde{B}_{0} e^{-\Omega t}$, with $\tilde{A}_{0}$ and $\tilde{B}_{0}$ fixed by the initial conditions. The second component of $\Phi(t), \Phi_{1}(t)$, can be deduced from $\Phi_{0}(t)$ since

$$
\Phi_{1}(t)=\frac{1}{\alpha \beta\left(\omega_{1}-\omega_{2}\right)}\left(\frac{1}{2}(\alpha+\beta)\left(\omega_{1}-\omega_{2}\right) \Phi_{0}(t)-(\alpha-\beta) \dot{\Phi}_{0}(t)\right) .
$$

Moreover

$$
\left\{\begin{array}{l}
\Phi_{2}(t)=A_{2} e^{-\left(\omega_{1}+\omega_{2}\right) t / 2}+\tilde{A}_{2} e^{\Omega t}+\tilde{B}_{2} e^{-\Omega t} \\
\Phi_{3}(t)=A_{3} e^{\left(\omega_{1}+\omega_{2}\right) t / 2}+\tilde{A}_{3} e^{\Omega t}+\tilde{B}_{3} e^{-\Omega t}
\end{array}\right.
$$

As for the asymptotic behavior of $\Psi(t)$, we recall that $\omega_{1}>\omega_{2}>0$. Of course, to conclude that $\Psi(t)=e^{-\Gamma t} \Phi(t) \rightarrow 0$ when $t \rightarrow \infty$, it is enough to consider the worse contribution, i.e. the one 
coming from $\Phi_{3}(t)$ : in fact, if this goes to zero, then it is clear that all the other components go to zero as well, so that $\|\Psi(t)\| \rightarrow 0$ when $t \rightarrow \infty$. We have, for $t \gg 1$,

$$
\Psi_{3}(t) \simeq \exp \left\{\left(\frac{1}{2}\left(\omega_{1}+\omega_{2}\right)-\Gamma\right) t\right\}=\exp \left\{\frac{t}{\alpha-\beta}\left(\alpha \omega_{2}-\beta \omega_{1}\right)\right\} .
$$

Recalling that $\alpha>\beta, \Psi_{3}(t) \rightarrow 0$ if and only if $\alpha \omega_{2}-\beta \omega_{1}<0$, i.e. if

$$
\frac{\alpha}{\beta}<\frac{\omega_{1}}{\omega_{2}}
$$

In other words, when condition (4.3) is satisfied, not only $\Psi_{3}(t)$, but $\Psi(t)$ itself decreases to zero for $t$ diverging.

\section{IV.2 Heisenberg representation}

Let us now consider the following operators

$$
\begin{aligned}
& a_{1}:=\frac{1}{\alpha}\left(\begin{array}{cccc}
\frac{-\beta^{2}}{\alpha-\beta} & \frac{\beta^{3}}{\alpha-\beta} & 0 & \beta \\
\frac{-\beta}{\alpha-\beta} & \frac{\beta^{2}}{\alpha-\beta} & 0 & 1 \\
\frac{\alpha^{2}-\beta}{\alpha-\beta} & \frac{\beta\left(-\alpha^{2}+\beta\right)}{\alpha-\beta} & 0 & 1 \\
0 & 0 & 0 & 0
\end{array}\right), \quad b_{1}:=\left(\begin{array}{cccc}
\frac{1}{\alpha-\beta} & \frac{-\alpha}{\alpha-\beta} & 1 & 0 \\
\frac{1}{\alpha(\alpha-\beta)} & \frac{-1}{\alpha-\beta} & \frac{1}{\alpha} & 0 \\
0 & 0 & 0 & 0 \\
\frac{-\alpha^{2}+\beta}{\alpha(\alpha-\beta)} & \frac{\alpha^{2}-\beta}{\alpha-\beta} & \frac{\beta}{\alpha} & 0
\end{array}\right), \\
& a_{2}:=\left(\begin{array}{cccc}
\frac{\beta}{\alpha-\beta} & \frac{-\beta^{2}}{\alpha-\beta} & 0 & -1 \\
\frac{\beta}{\alpha(\alpha-\beta)} & \frac{-\beta^{2}}{\alpha(\alpha-\beta)} & 0 & -\frac{1}{\alpha} \\
\frac{-\alpha}{\alpha-\beta} & \frac{\alpha^{2}}{\alpha-\beta} & 0 & 0 \\
\frac{\beta^{2}}{\alpha(\alpha-\beta)} & \frac{-\beta^{3}}{\alpha(\alpha-\beta)} & 0 & -\frac{\beta}{\alpha}
\end{array}\right), b_{2}:=\left(\begin{array}{cccc}
\frac{\beta}{\alpha(\alpha-\beta)} & \frac{-\beta}{\alpha-\beta} & \frac{\beta}{\alpha} & 0 \\
\frac{1}{\alpha(\alpha-\beta)} & \frac{-1}{\alpha-\beta} & \frac{1}{\alpha} & 0 \\
\frac{1}{\alpha(\alpha-\beta)} & \frac{-1}{\alpha-\beta} & \frac{1}{\alpha} & 0 \\
\frac{-\alpha}{\alpha-\beta} & \frac{\alpha \beta}{\alpha-\beta} & 0 & 0
\end{array}\right) .
\end{aligned}
$$

They satisfy the following anticommutation rules: $\left\{a_{j}, b_{k}\right\}=\delta_{j, k} \mathbb{1}_{4}$, with $\left\{a_{j}, a_{k}\right\}=\left\{b_{j}, b_{k}\right\}=$ $0, j, k=1,23$. Therefore they are two-dimensional pseudo-fermions. $H$ can be written in terms of these operators as

$$
H=i\left(\omega_{1} b_{1} a_{1}+\omega_{2} b_{2} a_{2}-\frac{\omega_{1}+\omega_{2}}{2} \mathbb{1}_{4}\right)
$$

As in the previous example, the time evolution of a given operator $X$, can be written in a way which extends (3.4):

$$
X_{e f f}(t)=e^{i H_{e f f}^{\dagger} t} X e^{-i H_{e f f} t}=e^{-\left(2 \Gamma+\omega_{1}+\omega_{2}\right) t} e^{\left(\omega_{1} \mathfrak{N}_{1}+\omega_{2} \mathfrak{N}_{2}\right) t} X e^{\left(\omega_{1} N_{1}+\omega_{2} N_{2}\right) t},
$$

\footnotetext{
${ }^{3}$ Other examples of $4 \times 4$ matrices satisfying these rules can be found in 23 .
} 
where $N_{j}=b_{j} a_{j}$ and $\mathfrak{N}_{j}=N_{j}^{\dagger}, j=1,2$. Recalling that $\left[N_{1}, N_{2}\right]=0$ and that $N_{j}^{2}=N_{j}$, we get

$$
N_{1, \text { eff }}(t)=e^{-\left(2 \Gamma+\omega_{2}\right) t}\left(N_{1}+\mathfrak{N}_{1} N_{1}\left(e^{\omega_{1} t}-1\right)\right)\left(\mathbb{1}_{4}+\left(N_{2}+\mathfrak{N}_{2}\right)\left(e^{\omega_{2} t}-1\right)+\mathfrak{N}_{2} N_{2}\left(e^{\omega_{2} t}-1\right)\right),
$$

and a similar expression can be deduced for $N_{2, \text { eff }}(t)$. Moreover, $\mathfrak{N}_{j, \text { eff }}(t)$ is simply the adjoint of $N_{j, e f f}(t)$. Using $\left\|N_{j}\right\| \leq 1$ and $\left\|\mathfrak{N}_{j}\right\| \leq 1, j=1,2$, we get

$$
\left\|N_{1, e f f}(t)\right\| \leq \exp \left\{-\left(2 \Gamma-\omega_{1}-\omega_{2}\right) t\right\},
$$

which goes to zero, when $t \rightarrow \infty$, if and only if $2 \Gamma>\omega_{1}+\omega_{2}$, i.e., if condition (4.3) is satisfied: we recover exactly (and not surprisingly) the same conclusion as in the Schrödinger representation.

Remark:- It might be interesting to notice that the matrices $a_{j}$ and $b_{j}$ above can be related to the following, standard, two-dimensional fermion annihilation operators,

$$
A_{1}=\left(\begin{array}{cccc}
0 & 1 & 0 & 0 \\
0 & 0 & 0 & 0 \\
0 & 0 & 0 & 1 \\
0 & 0 & 0 & 0
\end{array}\right), \quad A_{2}=\left(\begin{array}{cccc}
0 & 0 & 1 & 0 \\
0 & 0 & 0 & -1 \\
0 & 0 & 0 & 0 \\
0 & 0 & 0 & 0
\end{array}\right),
$$

as in [16]: $a_{j}=T A_{j} T^{-1}, b_{j}=T A_{j}^{\dagger} T^{-1}, j=1,2$, where $T$ is the following non-singular matrix:

$$
T=\left(\begin{array}{llll}
0 & \alpha & \beta & 0 \\
0 & 1 & 1 & 0 \\
\alpha & 0 & 1 & 0 \\
0 & \beta & 0 & \alpha
\end{array}\right)
$$

Here $\alpha \neq 0$ and $\alpha \neq \beta$. Therefore, this example can be considered as an explicit realization of the equivalence theorem stated in [16], concerning the relations between fermions and pseudofermions.

\section{An abstract generalization}

We will now describe how the examples considered in the previous sections can be further extended.

Let us consider two sets of pseudo-fermionic operators, $a_{j}$ and $b_{j}$, with $b_{j} \neq a_{j}^{\dagger}, j=$ $1,2, \ldots, N$, satisfying

$$
\left\{a_{j}, b_{k}\right\}=\delta_{j, k} \mathbb{1}, \quad\left\{a_{j}, a_{k}\right\}=\left\{b_{j}, b_{k}\right\}=0
$$


for all $j$ and $k$, and let $\varphi_{0,0, \ldots, 0}$ and $\Psi_{0,0, \ldots, 0}$, with $\left\langle\varphi_{0,0, \ldots, 0}, \Psi_{0,0, \ldots, 0}\right\rangle=1$, be the vacua of the $a_{j}$ 's and $b_{j}^{\dagger}$ 's respectively:

$$
a_{j} \varphi_{0,0, \ldots, 0}=b_{j}^{\dagger} \Psi_{0,0, \ldots, 0}=0,
$$

for all $j$. Then, extending the procedure of Section II, the sets $\mathcal{F}_{\varphi}$ and $\mathcal{F}_{\Psi}$ of the functions

$$
\varphi_{\underline{n}}:=\varphi_{n_{1}, n_{2}, \ldots, n_{N}}=b_{1}^{n_{1}} b_{2}^{n_{2}} \cdots b_{N}^{n_{N}} \varphi_{0,0, \ldots, 0},
$$

and

$$
\Psi_{\underline{n}}:=\Psi_{n_{1}, n_{2}, \ldots, n_{N}}=\left(a_{1}^{\dagger}\right)^{n_{1}}\left(a_{2}^{\dagger}\right)^{n_{2}} \cdots\left(a_{N}^{\dagger}\right)^{n_{N}} \Psi_{0,0, \ldots, 0},
$$

$n_{j}=0,1$, are biorthonormal bases of the $2^{N}$-dimensional Hilbert space $\mathcal{H}_{N}$.

Let now $i \dot{\Psi}(t)=H_{e f f} \Psi(t)$, with $H_{e f f} \neq H_{e f f}^{\dagger}$, be our original Schrödinger equation. As in Sections III and IV, we introduce a new wave function, $\Phi(t)=e^{\Gamma t} \Psi(t)$, where $\Gamma$ is a real constant, to be fixed. The Schrödinger equation for $\Phi(t)$ is

$$
i \dot{\Phi}(t)=H_{N} \Phi(t), \quad H_{N}:=H_{e f f}+i \Gamma \mathbb{1}_{N},
$$

where $\mathbb{1}_{N}$ is the identity operator on $\mathcal{H}_{N}$. The examples discussed before show that, in some interesting situations, the hamiltonian $H_{N}$ has the following general form:

$$
H_{N}=\sum_{j=1}^{N} \Omega_{j} N_{j}-\frac{1}{2} \sum_{j=1}^{N} \Omega_{j} \mathbb{1}_{N}
$$

where $N_{j}=b_{j} a_{j}$ is the $j$-th pseudo-fermionic number operator, while the $\Omega_{j}$ 's are, in general, complex quantities. Let us now define the following quantity:

$$
T_{N}:=\sum_{\underline{k}, \underline{n}}\left\langle\Psi_{\underline{n}}, H_{N} \varphi_{\underline{k}}\right\rangle=\sum_{\underline{k}}\left\langle\Psi_{\underline{k}}, H_{N} \varphi_{\underline{k}}\right\rangle,
$$

where the last equality is a consequence of the orthonormality of $\mathcal{F}_{\varphi}$ and $\mathcal{F}_{\Psi}$, and of the fact that $\varphi_{\underline{k}}$ is eigenstate of $H_{N}$. $T_{N}$ appears a natural extension of the trace of an operator to the present settings, where a single orthonormal basis is replaced by two biorthonormal bases. From (5.1) it is now easy to check that $T_{N}=0$ for all $N=1,2, \ldots$

In the (extended) Heisenberg representation, see Section 【I.1, the time evolution $X_{\text {eff }}(t)=$ $e^{i H_{e f f}^{\dagger} t} X e^{-i H_{e f f} t}$ of a given operator $X$, assume the following form:

$$
X_{e f f}(t)=e^{-t\left(2 \Gamma+\Im\left(\Omega_{1}\right)+\cdots+\Im\left(\Omega_{N}\right)\right)} \prod_{j=1}^{N} e^{i t \overline{\Omega_{j}} \mathfrak{N}_{j}} X \prod_{l=1}^{N} e^{i t \Omega_{l} N_{l}} .
$$


Focusing now to the time behavior of the number operator $N_{k}$, and recalling that $N_{j}^{2}=N_{j}$ and $\mathfrak{N}_{j}^{2}=\mathfrak{N}_{j}$, for all $j$, we deduce that

$$
\left(N_{k}\right)_{e f f}(t)=e^{-t\left(2 \Gamma+\Im\left(\Omega_{1}\right)+\cdots+\Im\left(\Omega_{N}\right)\right)} \prod_{j=1}^{N}\left(\mathbb{1}_{N}+\mathfrak{N}_{j}\left(e^{i t \overline{\Omega_{j}}}-1\right)\right) N_{k} \prod_{l=1}^{N}\left(\mathbb{1}_{N}+N_{l}\left(e^{-i t \Omega_{l}}-1\right)\right)
$$

Damping can be found by estimating the norm of the various $\left(N_{k}\right)_{e f f}(t)$. For that, we first recall that $\left\|N_{j}\right\|=\left\|\mathfrak{N}_{j}\right\| \leq 1$, for all $j$. The simplest situation is when the complex frequencies are all real: in fact, if $\Im\left(\Omega_{j}\right)=0$ for all $j$, we easily conclude that

$$
\left\|\left(N_{k}\right)_{e f f}(t)\right\| \leq 3^{2 N} e^{-2 \Gamma t}
$$

which goes to zero when $t$ diverges for all possible values of positive $\Gamma$. Hence, in this case, damping is deduced. It is probably more interesting to consider the situation in which not all the $\Omega_{j}$ 's are strictly real. In particular, we consider here the opposite situation, i.e. the one in which $\Im\left(\Omega_{j}\right) \neq 0$ for all $j$, since this is the most dangerous case. Now, the estimate above should be replaced by the following:

$$
\left\|\left(N_{k}\right)_{e f f}(t)\right\| \leq e^{-\left(2 \Gamma+\Im\left(\Omega_{1}\right)+\cdots+\Im\left(\Omega_{N}\right)\right) t} \prod_{j=1}^{N}\left(2+e^{t \Im\left(\Omega_{j}\right)}\right)^{2},
$$

which, clearly, extends the previous one. In this case, damping is recovered if

$$
\Gamma>\frac{1}{2} \sum_{j=1}^{N}\left|\Im\left(\Omega_{j}\right)\right|
$$

while a similar (and simpler) condition is recovered if only some of the $\Omega_{j}$ 's are not purely real. Notice that the inequality (5.4) becomes quite simple, $\Gamma>0$, when $\Im\left(\Omega_{j}\right)=0$, for all $j$. In this case, we recover our previous conclusion.

If, in analogy with $T_{N}$, we introduce the generalized trace also for $H_{\text {eff }}$, recalling that $T_{N}=0$ we find that

$$
T_{N}^{e f f}:=\sum_{\underline{k}}\left\langle\Psi_{\underline{k}}, H_{e f f} \varphi_{\underline{k}}\right\rangle=-i 2^{N} \Gamma .
$$

Therefore, in order for $H_{\text {eff }}$ to produce damping, the following inequality should be satisfied by the effective hamiltonian $H_{\text {eff }}$ :

$$
i T_{N}^{e f f}>2^{N-1} \sum_{j=1}^{N}\left|\Im\left(\Omega_{j}\right)\right| .
$$


We end this section with some remarks on the pseudo-fermionic structure related to this abstract system. The starting point is the pair of operators $S_{\varphi}$ and $S_{\Psi}$ which extends those of (2.4). They satisfy a multi-dimensional version of (2.10),

$$
N_{j} S_{\varphi}=S_{\varphi} \mathfrak{N}_{j}, \quad \mathfrak{N}_{j} S_{\Psi}=S_{\Psi} N_{j}
$$

for all $j$. Moreover, since the square root of both $S_{\varphi}$ and $S_{\Psi}$ can be defined, we can also introduce the self-adjoint operators $n_{j}=S_{\Psi}^{1 / 2} N_{j} S_{\varphi}^{1 / 2}=n_{j}^{\dagger}$. Therefore, if the $\Omega_{j}$ are real, we deduce that $S_{\Psi} H_{N}=H_{N}^{\dagger} S_{\Psi}$ and that

$$
h:=S_{\Psi}^{1 / 2} H_{N} S_{\varphi}^{1 / 2}=\sum_{j=1}^{N} \Omega_{j} n_{j}-\frac{1}{2} \sum_{j=1}^{N} \Omega_{j} \mathbb{1}_{N}
$$

is a self-adjoint operator.

It is well known that the operator $S_{\Psi}^{1 / 2}$, as well as its inverse $S_{\varphi}^{1 / 2}$, can be used to define a different scalar product in $\mathcal{H}_{N}$. Then the conclusion is the following: a damping effect in $\mathcal{H}_{N}$, endowed with its natural scalar product $<.$, . $>$, can be obtained using certain non self-adjoint effective hamiltonians which, when considered again in $\mathcal{H}_{N}$, but endowed with a different scalar product, are self-adjoint. In fact, let us define a new scalar product $\langle.,\rangle_{\Psi}$ on $\mathcal{H}_{N}$ as follows:

$$
\langle f, g\rangle_{\Psi}:=\left\langle S_{\Psi}^{1 / 2} f, S_{\Psi}^{1 / 2} g\right\rangle
$$

for all $f, g \in \mathcal{H}_{N}$. Then, since the intertwining relation above implies that $H_{N}=S_{\Psi}^{-1} H_{N}^{\dagger} S_{\Psi}$, we get

$$
\begin{aligned}
\left\langle f, H_{N} g\right\rangle_{\Psi} & =\left\langle S_{\Psi}^{1 / 2} f, S_{\Psi}^{1 / 2} H_{N} g\right\rangle=\left\langle S_{\Psi} S_{\Psi}^{-1} H_{N}^{\dagger} S_{\Psi} f, g\right\rangle= \\
& =\left\langle S_{\Psi}^{1 / 2} H_{N} f, S_{\Psi}^{1 / 2} g\right\rangle=\left\langle H_{N} f, g\right\rangle_{\Psi},
\end{aligned}
$$

for all $f, g \in \mathcal{H}_{N}$. Hence $H_{N}$ turns out to be self-adjoint with respect to $\langle.,\rangle_{\Psi}$.

Alternatively (and more straightforwardly), formula (5.5) shows that the effective hamiltanians considered here and producing damping are similar (but not unitarily equivalent) to self adjoint operators. A natural and quite interesting question to consider is therefore: is this a general requirement? We have no answer now, but this is part of our works in progress.

\section{Conclusions}

After some general remarks on the time evolution driven by a non self-adjoint hamiltonian, we have shown how this can be used in the analysis of the decay of finite dimensional systems 
in connection with pseudo-fermions. In particular, in the examples discussed here, we have seen that the effective hamiltonians describing damping are related, in a very direct way, to pseudo-hermitian operators. We have also shown that the Heisenberg representation can be conveniently adopted in the analysis of the time evolutions of these systems.

The next natural step of our research will be to check how much of this approach can be extended to infinite dimensional spaces and to try to answer to the following, rather general, question: can any effective damping be described in terms of some pseudo-hermitian hamiltonian?

\section{Acknowledgements}

The author acknowledges financial support by the MIUR.

\section{References}

[1] F. Bagarello, Pseudo-bosons, Riesz bases and coherent states, J. Math. Phys., 50, DOI:10.1063/1.3300804, 023531 (2010) (10pg)

[2] F. Bagarello Construction of pseudo-bosons systems, J. Math. Phys., 51, doi:10.1063/1.3300804, 023531 (2010) (10pg)

[3] F. Bagarello Mathematical aspects of intertwining operators: the role of Riesz bases, J. Phys. A, doi:10.1088/1751-8113/43/17/175203, 43, 175203 (2010) (12pp)

[4] F. Bagarello, F. Calabrese Pseudo-bosons arising from Riesz bases, Bollettino del Dipartimento di Metodi e Modelli Matematici, 2, 15-26, (2010)

[5] F. Bagarello, (Regular) pseudo-bosons versus bosons, J. Phys. A, 44, 015205 (2011)

[6] F. Bagarello, Examples of Pseudo-bosons in quantum mechanics, Phys. Lett. A, 374, 38233827 (2010)

[7] S.T. Ali, F. Bagarello, J.-P. Gazeau, Modified Landau levels, damped harmonic oscillator and two-dimensional pseudo-bosons, J. Math. Phys., 51, 123502 (2010)

[8] F. Bagarello, Pseudo-bosons, so far, Rep. Math. Phys., 68, No. 2, 175-210 (2011) 
[9] D.A. Trifonov, Pseudo-boson coherent and Fock states, arXiv: quant-ph/0902.3744, Differential Geometry, Complex Analysis and Mathematical Physics, eds. K. Sekigawa et al (W. Scientific 2009), pp. 241-250

[10] F. Bagarello, Non linear pseudo-bosons, J. Math. Phys., J. Math. Phys., 52, 063521, (2011)

[11] F. Bagarello, M. Znojil, Non linear pseudo-bosons versus hidden Hermiticity, J. Phys. A, 44 415305, (2011)

[12] F. Bagarello, M. Znojil, Non linear pseudo-bosons versus hidden Hermiticity. II: The case of unbounded operators, J. Phys. A, 45, 115311 doi:10.1088/1751-8113/45/11/115311, (2012)

[13] C. Bender, Making Sense of Non-Hermitian Hamiltonians, Rep. Progr. Phys., 70, 947-1018 (2007)

[14] A. Mostafazadeh, Pseudo-Hermitian representation of Quantum Mechanics, Int. J. Geom. Methods Mod. Phys. 7, 1191-1306 (2010)

[15] M. Znojil, Three-Hilbert-space formulation of Quantum Mechanics, SIGMA 5, 001 (2009)

[16] F. Bagarello, Linear pseudo-fermions, J. Phys. A, in press

[17] O. Cherbal, M. Drir, M. Maamache, D. A. Trifonov, Fermionic coherent states for pseudoHermitian two-level systems, J. Phys. A, 40, 1835-1844, (2007)

[18] A. Mostafazadeh, Pseudo-Hermitian representation of Quantum Mechanics, J. Phys. A, 37 10193-10207, (2004)

[19] C. M. Bender, S. P. Klevansky, PT-symmetric representations of fermionic algebras, Phys. Rev. A, 84, 024102 (2011)

[20] Y. Ben-Aryeh, A. Mann, I. Yaakov, Rabi oscillations in a two-level atomic system with a pseudo-hermitian hamiltonian, J. Phys. A, 37 12059-12066, (2004)

[21] E.-M. Graefe, M. Hoening, H. J. Korsch, Classical limit of non-Hermitian quantum dynamics-a generalized canonical structure, J. Phys. A, 43, 075306, (2010)

[22] F. Bagarello, M. Znojil, The dynamical problem for a non self-adjoint hamiltonian, Proceedings of IWOTA 2011 (July 1216, 2010, Berlin, Germany), Operator Theory: Advances and Applications, 221, 109 - 119, 2012 
[23] O. Cherbal, D. A. Trifonov, Extended PT- and CPT-symmetric representations of fermionic algebras, Phys. Rev. A 85, 052123 (2012) 